Fundación

Miguel Lillo

Tucumán

Argentina

\title{
Estructura y composición de las bandadas mixtas de aves invernales a lo largo del gradiente latitudinal en las selvas montanas de las Yungas, Argentina
}

\author{
Structure and composition of winter mixed species birds flocks \\ along the latitudinal gradient of the subtropical montane forest of \\ the Yungas, Argentina
}

María Elisa Fanjul 1,2*, Ada Echevarria 1, María Valeria Martínez ${ }^{1}$

1 Instituto de Vertebrados, Área de Zoología, Fundación Miguel Lillo. Miguel Lilo 251, (4000) San Miguel de Tucumán, Argentina.

2 Facultad de Ciencias Naturales e IML - UNT. Miguel Lillo 205, (4000) San Miguel de Tucumán, Argentina.

*mefanjul@lillo.org.ar

\section{RESUMEN}

Las bandadas mixtas (BM) son asociaciones de diferentes especies que se forman como estrategia para aumentar la eficiencia de forrajeo y/o reducir el riesgo de predación. El objetivo del presente trabajo fue determinar la variación en la composición y estructura de las BM, a lo largo del gradiente latitudinal de la selva montana de las Yungas argentinas. Ocho sitios fueron estudiados a lo largo de $105700 \mathrm{~km}$ de distribución de las Yungas en el país. En cada sitio se establecieron 10 transectas visitadas de junio a septiembre entre 2006 y 2009. Se determinó riqueza y abundancia de las BM. Se observaron $325 \mathrm{BM}$, con un total de 72 especies participantes y una abundancia de 2480 individuos. Diecisiete familias estuvieron representadas, 15 del Orden Passeriformes y 2 no-Passeriformes (Trochilidae y Picidae). Las familias más importantes fueron Tyrannidae (12 especies), Furnariidae (10 especies) y Thraupidae (nueve especies). Doce especies fueron omnipresentes en los ocho sitios de estudio y las especies que tuvieron los valores de ocurrencia más altos fueron: Syndactyla rufosuperciliata, Phylloscartes ventralis, Chlorospingus flavopectus y Myioborus brunniceps. Aunque los análisis estadísticos multivariados indicaron que el gradiente latitudinal no tendría influencia directa sobre las características estructurales (número de ban-

Ref. bibliográfica: Fanjul, M. E.; Echevarria, A.; Martínez, M. V. 2021. “Estructura y composición de las bandadas mixtas de aves invernales a lo largo del gradiente latitudinal en las selvas montanas de las Yungas, Argentina". Acta zoológica lilloana 65 (2): 268-286. doi: https://doi.org/10.30550/j.azl/2021.65.2/2021-11-04 > Recibido: 22 de septiembre 2021 - Aceptado: 4 de noviembre 2021.

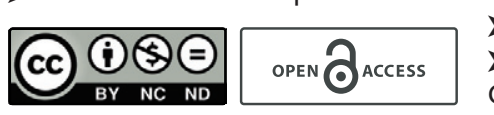

- URL de la revista: http://actazoologica.lillo.org.ar

- Esta obra está bajo una Licencia Creative Commons Atribución - No Comercial - Sin Obra Derivada 4.0 Internacional. 
dadas, número de especies e individuos involucrados) de las BM, la composición de especies varió en asociación con la latitud. Algunas causas asociadas al gradiente, como el clima local, características de la vegetación y disponibilidad de alimento, podrían influir en los resultados obtenidos.

Palabras clave - Gradientes ambientales, avifauna, agrupación mixta, selva subtropical.

\begin{abstract}
Mixed-species flocks (MSF) are associations of different species formed as a strategy to increase the efficiency of foraging and to reduce the risk of predation. The aim of the present study was to determine the variation of the composition and structure of the MSF, along a latitudinal gradient in the subtropical mountain forest of the Yungas of Argentina. Eight sampling sites were studied along the $700 \mathrm{~km}$ of distribution of the Yungas in Argentina. Ten transects at each site were established and were visited from June to September between 2006 and 2009. It was determined the richness and abundance of the MSF. 325 MSF, with a total of 72 participating species and an abundance of 2480 individuals were observed. Seventeen families were represented, 15 of the order Passeriformes and two non-passerines (Trochilidae and Picidae). Tyrannidae (12 species), Furnariidae (10 species) and Thraupidae (nine species) were the major families. Twelve species were omnipresent in the eight study sites, being Syndactyla rufosuperciliata, Phylloscartes ventralis, Chlorospingus flavopectus and Myioborus brunniceps the species that had the highest values of occurrence. Although multivariate statistical analyzes, indicated that the latitudinal gradient would have no direct influence on the structural characteristics (number of flocks, number of species and individuals involved) of the MSF, the composition of species varied in association with latitude. Some causes associated with the gradient, such as local climate, vegetation characteristics and food availability, could influence the results obtained.
\end{abstract}

Palabras clave - Environment gradients, birdlife, mixed groups, subtropical mountain forest.

\title{
INTRODUCCIÓN
}

Un fenómeno biológico llamativo de organización social en animales silvestres es el de los grupos heteroespecíficos, definidos como asociaciones compuestas por individuos de diferentes especies, que se desplazan y se alimentan juntos (Moynihan, 1962; Terborgh, 1990; Sridhar et al. 20012; Goodale et al. 2020). Estos grupos han sido documentados para numerosos taxones de vertebrados, principalmente peces (Semeniuk y Dill, 2006; Sazima, Krajewski, Bonaldo, Sazima, 2007), aves (Moynihan, 1962; Goodale, Beauchamp, Magrath, Nieh, Ruxton, 2010) y mamíferos (Struhsaker, 1981; Stensland, Angerbjörn, Berggren, 2003; Stojan-Dolar y Heymann, 2010). Las bandadas mixtas de aves se definen como asociaciones de individuos, parejas o grupos de diferentes especies que se desplazan y alimentan juntas (Morse, 1970; Munn y Terborgh, 1979). 
En las bandadas mixtas, las especies desempeñan diferentes roles y reciben variados beneficios de dicha asociación (Munn, 1983). En general, se plantean dos hipótesis acerca de los beneficios de formación de bandadas mixtas, las cuales no son mutuamente exclusivas (Goodale et al., 2020). Las aves participan en bandadas mixtas y de esa forma aumentan su eficiencia de forrajeo (Moynihan, 1962; Morse, 1977; Munn y Terborgh, 1979; Powell, 1985; King y Rappole, 2000) y/o aumentan la detección de predadores (Moynihan, 1962; Colorado, 2013; Zou et al., 2018).

La mayoría de las investigaciones de ecología de bandadas mixtas realizadas en la región Neotropical, fueron en ambientes tropicales y en menor medida en regiones subtropicales y templadas (King y Rappole, 2000; Pomara, Cooper, Petit, 2003; Tierno de Figueroa y Padial, 2005; Tubelis, 2007; Amaral y Ragusa-Netto, 2008; Knowlton y Graham, 2011; Mangini y Fanjul, 2013; Zarco y Cueto 2017; Kajiki, Montaño-Centellas, Mangini, Colorado, Fanjul, 2018; Zarco, Cueto, Sagario y Marone 2019; Montaño-Centellas 2020). En general en estas regiones, las bandadas mixtas se forman principalmente durante el otoño e invierno (época no reproductiva), cuando las condiciones climáticas son desfavorables y el recurso alimenticio generalmente es escaso; por esto, las mismas tienden a desaparecer o ser menos activas en la época reproductiva (Maldonado-Coelho y Marini, 2000; Ippi y Trejo, 2003; Martínez, 2003; Tubelis, Cowling, Donnelley, 2006; Fanjul y Echevarria, 2015; Mangini y Areta, 2018). Como se ha evidenciado en otros estudios, la estructura y composición de las bandadas mixtas están íntimamente relacionadas con el tipo de ambiente donde interactúan (Mokross, Ryder, Côrtes, Wolfe, Stouffer, 2014; Goodale et al., 2015, Kajiki et al., 2018; Montaño-Centellas, 2020), con variantes entre regiones, localidades y hábitats (Powell, 1985). Aunque existe un recambio de individuos y especies dentro de una bandada a través del espacio y del tiempo, la mayoría de los miembros que participan lo seguirán haciendo a menos que el ambiente se vea alterado (Martínez, 2003; Zhang, Han, Huang y Zou, 2013; Borah, Quader, Srinivasan, 2018).

Las selvas subtropicales de Las Yungas en Argentina, se extienden desde el norte de Salta (en el límite con Bolivia) hasta el norte de la provincia de Catamarca, a lo largo de un gradiente latitudinal norte-sur de aproximadamente $700 \mathrm{~km}$ de distribución. En diferentes taxones, como aves (Nores, 1989; Vides-Almonacid, 1993; Blendinger y Álvarez, 2009), mamíferos (Ojeda, Barquez, Stadler, Brandl, 2008) y árboles (Morales, Sirombra, Brown, 1995; Blundo, Malizia, Blake, Brown, 2012) se puede observar un patrón de disminución de la diversidad a lo largo del gradiente latitudinal, el cual estaría relacionado fundamentalmente, a un empobrecimiento climático latitudinal, producido por la disminución de la temperatura y las precipitaciones que influyen en la estructura de la vegetación local (Brown, Grau, Malizia, Grau, 2001; Ojeda et al., 2008; Blundo et al., 2012).

Considerando el potencial efecto del gradiente latitudinal sobre algunas características de las aves como, por ejemplo, riqueza y abundancia, resulta interesante determinar si dicho efecto influye también sobre la composición y estructura de las bandadas mixtas invernales en las Yungas de Argentina. El en presente trabajo, el objetivo es, evaluar cómo varía la estructura y composición de las bandadas mixtas a lo largo de la selva montana de las Yungas de Argentina, durante períodos invernales. 


\section{MATERIALES Y MÉTODOS}

\section{Área de estudio}

En Argentina, las Yungas, se extienden por las laderas orientales de los Andes, sobre las Sierras Subandinas y las Sierras Pampeanas desde el límite con Bolivia, en el norte de Salta, hasta el norte de Catamarca. Se distribuyen a lo largo de un gradiente latitudinal, en sentido Norte-Sur, sobre una angosta franja de aproximadamente 50 $\mathrm{km}$ de ancho y unos $700 \mathrm{~km}$ de longitud, atravesando las provincias de Jujuy, Salta, Tucumán y Catamarca, en un rango altitudinal que va desde los 350 a los $3000 \mathrm{~m}$ s.n.m. (Cabrera, 1976; Vervoorst, 1979; Brown et al., 2001) (Figura 1). Las selvas montanas (entre los 550 a $1600 \mathrm{~m}$ s.n.m.) se caracterizan por ser el piso altitudinal de máxima humedad dentro de las Yungas, con precipitaciones anuales totales entre 1500 y $3000 \mathrm{~mm}$ y por ser el sitio más diverso, tanto en especies vegetales como animales. Las especies vegetales dominantes son de origen tropical, con una gran riqueza de leñosas tales como: horco molle (Blepharocalyx gigantea), laurel (Phoebe porphyiria, Ocotea puberula), cedro (Cedrela lilloi), tipa blanca (Tipuana tipu), nogal (Fuglans australis), arrayán (Eugenia uniflora), entre otras (Morales et al., 1995; Brown et al., 2001). En cuanto a la fauna, se puede mencionar entre los vertebrados a un importante número de endemismos en los anfibios, entre los que se encuentran la ranita de la hojarasca tucumana (Oreobates discoidales) y sapito panza roja de la Yunga (Melanophryniscus rubriventris); o la presencia de mamíferos con importantes poblaciones, como ser, Yaguareté (Panthera onca), Tapir terrestre (Tapirus terrestris) y Pecarí labiado (Tayassu pecari) (Brown et al., 2001).

En el presente estudio, se dividió a las selvas montanas en tres sectores: Sector Norte, Sector Centro y Sector Sur, abarcando una extensión desde los $22^{\circ} \mathrm{S}$ a los $28^{\circ} \mathrm{S}$ (Figura 1), en los cuales se ubicaron ocho sitios de estudios. Dicha división estuvo basada en los criterios de Brown et al. 2001 y Malizia, Pacheco, Blundo y Brown 2012. Todos los sitios se encontraban entre los 800 y 1200 m s.n.m. y se caracterizaron por estar en buen estado de conservación, principalmente dentro de áreas protegidas. Los sitios fueron los siguientes: Sector Norte: Baritú $\left(22^{\circ} 37^{\prime} S-64^{\circ} 37^{\prime} \mathrm{O}\right.$,

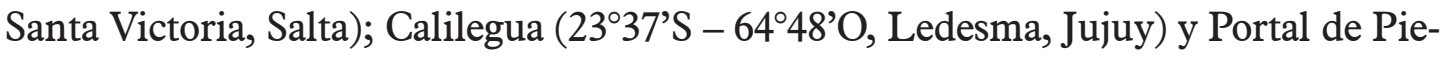
dras ( $24^{\circ} 05^{\prime} \mathrm{S}-64^{\circ} 24^{\prime} \mathrm{O}$, Santa Bárbara, Jujuy). Sector Centro: Las Maderas (24⒉

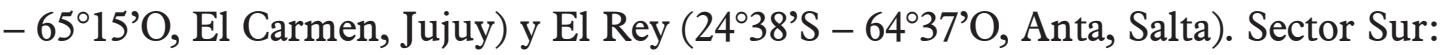
Sierra de San Javier (26 $47^{\prime} \mathrm{S}-65^{\circ} 20^{\prime} \mathrm{O}$, Yerba Buena, Tucumán); Campo de los Alisos $\left(27^{\circ} 20^{\prime} \mathrm{S}-65^{\circ} 46^{\prime} \mathrm{O}\right.$, Concepción, Tucumán) e Higuerillas ( $27^{\circ} 45^{\prime} \mathrm{S}-65^{\circ} 48^{\prime} \mathrm{O}$, Escaba, J.B. Alberdi, Tucumán).

\section{Métodos}

Aves.- El trabajo se llevó a cabo desde mayo de 2006 hasta septiembre de 2009, durante las estaciones de otoño/invierno. En cada sitio se ubicaron 10 transectas de faja de $300 \mathrm{~m}$ de largo por $20 \mathrm{~m}$ de ancho, visitadas tres veces en las primeras horas de la mañana y tres en las últimas horas de la tarde durante un único año de muestreo cada sitio (Ralph et al., 1996; Bibby, Jones, Marsden, 2000). Se conside- 


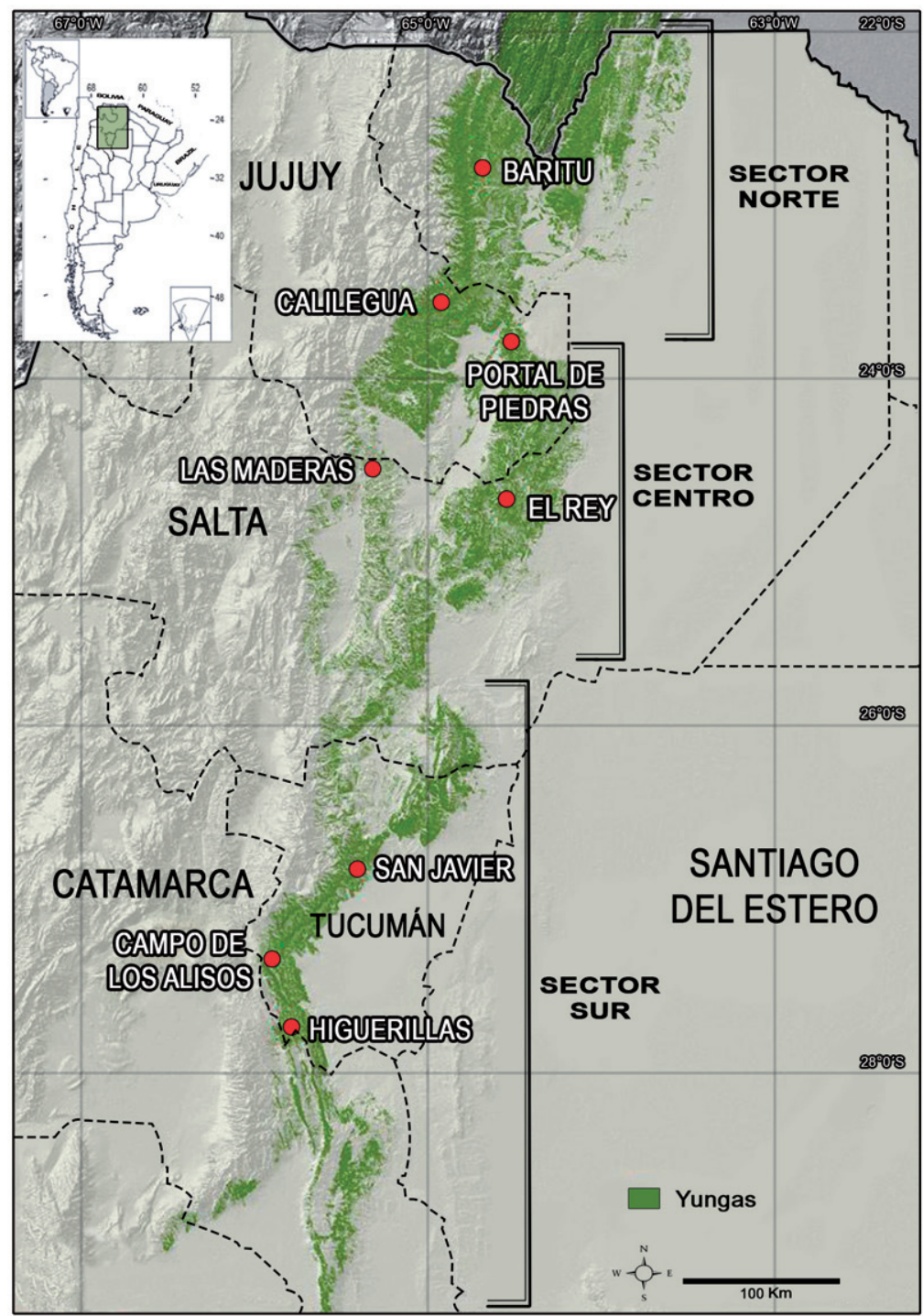

Figura 1. Distribución de las Yungas en Argentina y ubicación de los sitios de estudio. Las líneas indican los diferentes sectores de las Yungas. Figura modificada de Fundación ProYungas, http://siga. proyungas.org.ar/mapas/

Figure 1. Distribution of the Yungas in Argentina and location of study sites. The lines indicate the different sectors of the Yungas. Figure modified from Fundación ProYungas, http://siga.proyungas. org.ar/mapas/

ró bandada mixta a un grupo de dos o más individuos, de dos o más especies que aparecen juntas en una distancia máxima de $25 \mathrm{~m}$ de separación y que se mueven en la misma dirección (Hutto, 1987). Para el estudio de dichas bandadas, se siguió la metodología de Moynihan (1962), Morse (1970) y Jullien y Thiollay (1998). En cada oportunidad que se observó una bandada mixta, se la siguió por un período máximo de 10 minutos, aunque ésta se alejara de la transecta. Las observaciones se realizaron con binoculares $(10 \mathrm{x} 40 \mathrm{~mm})$ y en los casos de contactos con bandadas se especificaron: especies observadas y número de individuos por especie. La nomenclatura y clasificación taxonómica se realizó en base a los criterios de Pearman y Areta (2020). 
Análisis Estadísticos. - La ocurrencia de las especies en las bandadas mixtas por sitio, se determinó como el número de veces que cada especie participó dividido por el número total de bandadas mixtas observadas, expresado en porcentaje. La ocurrencia general de especies en las bandadas mixtas, se obtuvo como: el número de veces que cada especie participó dividido por el número total de bandadas mixtas observadas en todos los sitios de estudio. Se definieron cuatro categorías de ocurrencia: Baja: 0 a 25\%; Media: 26 a 50\%; Alta: 51 a 75\%; Muy alta: 76 a 100\% (Jullien y Thiollay, 1998; Cordeiro et al., 2015).

Para evaluar cómo varía el número de bandadas mixtas y el tamaño (número de especies e individuos por bandada mixta), a lo largo de los diferentes sitios de estudio, se realizaron Modelos Lineales Generalizados Mixtos (GLMM, en inglés) (Mokross et al., 2014, Cordeiro et al., 2015). Se utilizó un GLMM debido al efecto anidado de las transectas que pertenecen a un mismo sitio (Zuur, Ieno, Walker, Saveliev, Smith, 2009). Se consideraron los sitios de estudio y a los tres sectores (norte, centro y sur) como los interceptos aleatorios y como variables fijas. Se usaron medidas de distancia de Poisson (ya que son datos obtenidos por conteos). Se utilizaron diferentes modelos según las diferentes variables respuestas (número de bandadas mixtas, el número de especies por bandada mixta y número de individuos por bandada mixta). La selección del mejor modelo fue el que presentó el menor valor de criterio de Información de Akaike (Zuur et al., 2009). Los análisis fueron realizados en $\mathrm{R}$ usando el paquete 'nlme' ( $\mathrm{R}$ Development Core Team, 2004).

Para determinar la variación en la composición de especies de las bandadas a lo largo de los sitios se realizaron dos análisis. Un análisis numérico, el Índice de Similitud de Sorensen (Magurran, 1989), en el cual se utilizaron datos cualitativos de presencia/ausencia de las especies participantes en las bandadas mixtas en los diferentes sitios. En este índice, 1 indica similitud completa y 0 sin especies en común y los resultados se expresan en porcentaje. Y un análisis multivariado de ordenamiento y similitud, Nonmetric Multidimensional Scaling (NMDS en inglés). Este análisis representa de manera gráfica la similitud o disimilitud de una comunidad en relación a los sitios de estudio (McCune y Grace, 2002). Se utilizaron datos de presencia-ausencia como una medida de evaluar la riqueza, y datos de abundancia relativa de las especies que participaron en las bandadas mixtas. En el presente trabajo, el análisis recomendó graficar en dos dimensiones.

\section{RESULTADOS}

En este estudio se observaron 325 bandadas mixtas, pertenecientes a 72 especies y con una abundancia total de 2480 individuos. Diecisiete familias estuvieron representadas, 15 del Orden Passeriformes y dos no-Passeriformes (Trochilidae y Picidae). Las familias más importantes fueron: Tyrannidae (12 especies), Furnariidae (10 especies) y Thraupidae (nueve especies) (Apéndice 1). Aproximadamente el $50 \%$ de las bandadas mixtas registradas estuvieron compuestas de 2 a 7 especies y el $60 \%$ de las mismas tuvieron entre 3 a 10 individuos, variando entre los diferentes sitios. El mayor número de bandadas mixtas fue registrado en San Javier, como así también el 
mayor número de individuos participantes de bandadas mixtas. El mayor número de especies se registró en Las Maderas. En Portal de Piedras se encontró la bandada de mayor tamaño, con el mayor número de especies e individuos. En promedio, Portal de Piedra e Higuerillas presentaron los valores más altos de especies por bandada mixta y en Campo de los Alisos se registraron los valores más bajos en todas las variables mencionadas (Tabla 1 ).

Doce especies fueron omnipresentes en todos los sitios de estudios y son comunes en las selvas montanas de las Yungas: Sittasomus griseicapillus, Synallaxis azarae, Syndactyla rufosuperciliata, Phylloscartes ventralis, Mecocerculus leucophrys, Turdus rufiventris, Myioborus brunniceps, Setophaga pitiayumi, Thraupis sayaca, Microspingus erythrophrys, Arremon flavirostris y Chlorospingus flavopectus. Cuatro de ellas, presentaron los valores de ocurrencia más alto en las bandadas mixtas: Syndactyla rufosuperciliata, Phylloscartes ventralis y Chlorospingus flavopectus y Myioborus brunniceps, con valores entre 35 y 53\%. El resto de las especies tuvieron valores de ocurrencia por debajo del 30\% (Apéndice 1).

Los Modelos Lineales Generalizados Mixtos (GLMM) mostraron que la riqueza de especies dependió de la interacción entre los sitios de estudios ubicados de norte a sur y el número de bandadas mixtas registradas por sitio $(\mathrm{AIC}=431,63 ; \mathrm{BIC}=$ 443,15; $\log L i k=-210,82$, Tabla 2). Ningún modelo logró, de manera significativa, evidenciar relación entre las características estructurales de las bandadas mixtas a lo largo del gradiente. A nivel de sectores (norte, centro y sur), el número de bandadas mixtas varió significativamente de manera positiva con el número de individuos participantes, junto con una interacción significativa y negativa con la riqueza y el sector centro $(\mathrm{AIC}=259,34 ; \mathrm{BIC}=279,95 ; \log \mathrm{Lik}=-120,67$, Tabla 3 ).

La similitud en composición de especies entre los diferentes sitios según el índice de Sorensen registró valores entre 44 y 78\%. La mayor similitud se dio entre Baritú y Portal de Piedras (78,1\%), seguido por Calilegua y El Rey (73,5\%). Los sitios pertenecientes al sector Norte (Baritú, Calilegua y Portal de Piedras) mostraron una similitud del 70,13\%. Para el sector Centro (Las Maderas y El Rey) la similitud fue del 63,9\% mientras que para el sector Sur (San Javier, Campo de los Alisos e Higuerillas) la similitud fue de alrededor del $65 \%$.

El Análisis multivariado de similitud Nonmetric Multidimensional Scaling (NMDS) explicó el 71,03\% de la variabilidad de los datos de presencia-ausencia en sus dos primeros ejes, demostrando una similitud en la composición de especies entre los sitios (Estres Final = 26,31; Valor de Inestabilidad =0,001). Los sitios se disponen en relación al eje 1 de norte a sur a lo largo del gradiente de las Yungas y se evidencia una separación en los diferentes sectores (Figura 2 A). En función de la abundancia relativa entre sitios, el análisis indicó variabilidad en los dos primeros ejes de un 75, 02\% (Estres Final = 29,45; Inestabilidad Final = 0,03). Se puede observar que los sitios se disponen en relación al eje 2 de norte a sur a lo largo del gradiente de las Yungas y se evidencia la separación de los sectores (Figura 2 B). 
Tabla 1. Datos generales de las bandadas mixtas en los ocho sitios de estudio de las selvas montanas de las Yungas de Argentina.

Table 1. General data of the mixed-species flocks in the eight study sites of the subtropical montane forests of the Yungas of Argentina.

\begin{tabular}{|c|c|c|c|c|c|c|c|c|}
\hline & Baritú & Calilegua & $\begin{array}{l}\text { P. de } \\
\text { Piedras }\end{array}$ & $\begin{array}{l}\text { Las } \\
\text { maderas }\end{array}$ & El Rey & $\begin{array}{l}\text { San } \\
\text { Javier }\end{array}$ & Alisos & $\begin{array}{l}\text { Higue- } \\
\text { rillas }\end{array}$ \\
\hline $\mathrm{N}^{\circ}$ Bandadas Mixtas (BM) & 38 & 45 & 41 & 36 & 41 & 51 & 27 & 46 \\
\hline No Total Especies & 28 & 35 & 36 & 40 & 30 & 26 & 24 & 34 \\
\hline $\begin{array}{l}\text { Media especies / BM } \\
\text { y DE }\end{array}$ & $\begin{array}{c}3,71 \\
( \pm 1,75)\end{array}$ & $\begin{array}{c}4,31 \\
( \pm 1,86)\end{array}$ & $\begin{array}{c}5,29 \\
( \pm 2,98)\end{array}$ & $\begin{array}{c}4,97 \\
( \pm 2,89)\end{array}$ & $\begin{array}{c}4,49 \\
( \pm 2,08)\end{array}$ & $\begin{array}{c}4,53 \\
( \pm 2,48)\end{array}$ & $\begin{array}{c}3,30 \\
( \pm 1,27)\end{array}$ & $\begin{array}{c}5,11 \\
( \pm 2,73)\end{array}$ \\
\hline $\begin{array}{l}\text { Media individuos / BM } \\
\text { y DE }\end{array}$ & $\begin{array}{c}6,29 \\
( \pm 3,48)\end{array}$ & $\begin{array}{c}7,47 \\
( \pm 3,92)\end{array}$ & $\begin{array}{c}8,89 \\
( \pm 5,91)\end{array}$ & $\begin{array}{c}7,83 \\
( \pm 4,96)\end{array}$ & $\begin{array}{c}8,71 \\
( \pm 3,94)\end{array}$ & $\begin{array}{c}7,53 \\
( \pm 4,20)\end{array}$ & $\begin{array}{c}5,26 \\
( \pm 2,63)\end{array}$ & $\begin{array}{c}8,04 \\
( \pm 5,49)\end{array}$ \\
\hline $\mathrm{N}^{\circ}$ mínimo especies / BM & 2 & 2 & 2 & 2 & 2 & 2 & 2 & 2 \\
\hline $\mathrm{N}^{\circ}$ máximo especies / BM & 9 & 9 & 14 & 13 & 11 & 13 & 7 & 13 \\
\hline No mínimo individuos / BM & 2 & 2 & 3 & 2 & 2 & 2 & 2 & 2 \\
\hline No máximo individuos / BM & 17 & 17 & 29 & 18 & 18 & 21 & 12 & 25 \\
\hline $\mathrm{N}^{\circ}$ total individuos & 240 & 336 & 365 & 292 & 357 & 384 & 144 & 370 \\
\hline
\end{tabular}

Tabla 2. Resultados del Modelo Lineal Generalizado Mixto de la riqueza de especies de las bandadas mixtas en función de los sitios de estudio. Se muestran los efectos fijos (los sitios).

Table 2. Results of the Generalized Linear Mixed Model of species richness of mixed-species flocks as a function of study sites. Fixed effects (sites) are shown.

\begin{tabular}{l|c|c|c|c} 
& Valor & $\begin{array}{c}\text { Error } \\
\text { estándar }\end{array}$ & $\begin{array}{c}\text { Grados de } \\
\text { libertad }\end{array}$ & $T$ \\
\hline Intercepto & 10,45 & 1,03 & 68 & 10,13 \\
\hline Sitio & $-1,10$ & 0,25 & 6 & $-4,35$ \\
\hline Sitio: $N^{\circ}$ Bandadas Mixtas & 0,30 & 0,04 & 68 & 7,48 \\
\hline
\end{tabular}

Referencias: En negrita cursiva, las diferencias significativas $(P<0,05)$.

Tabla 3. Resultados del Modelo Lineal Generalizado Mixto del número de bandadas mixtas en función de los efectos fijos de los Sectores de estudio, y de las interacciones con las variables riqueza y número de individuos.

Table 3. Results of the Generalized Linear Mixed Model of the number of mixed-species flocks as a function of the fixed effects of the study Sectors, and of the interactions with the variables richness and number of individuals.

\begin{tabular}{l|c|c|c|c} 
& Valor & $\begin{array}{c}\text { Error } \\
\text { estándar }\end{array}$ & $\begin{array}{c}\text { Grados de } \\
\text { libertad }\end{array}$ & $T$ \\
\hline Intercepto & 0,60 & 0,40 & 68 & 5 \\
\hline Sector Centro & 0,90 & 0,65 & 5 & 1,49 \\
\hline Sector Sur & 0,16 & 0,68 & 1 & 0,23 \\
\hline Riqueza & 0,08 & 0,05 & 68 & 1,66 \\
\hline Abundancia & 0,09 & 0,01 & 68 & 7,95 \\
\hline Sector Centro: Riqueza & $-0,13$ & 0,05 & 68 & $-2,52$ \\
\hline Sector Sur: Riqueza & $-0,01$ & 0,05 & $-0,28$ \\
\hline
\end{tabular}

Referencias: En negrita cursiva, las diferencias significativas $(P<0,05)$. 

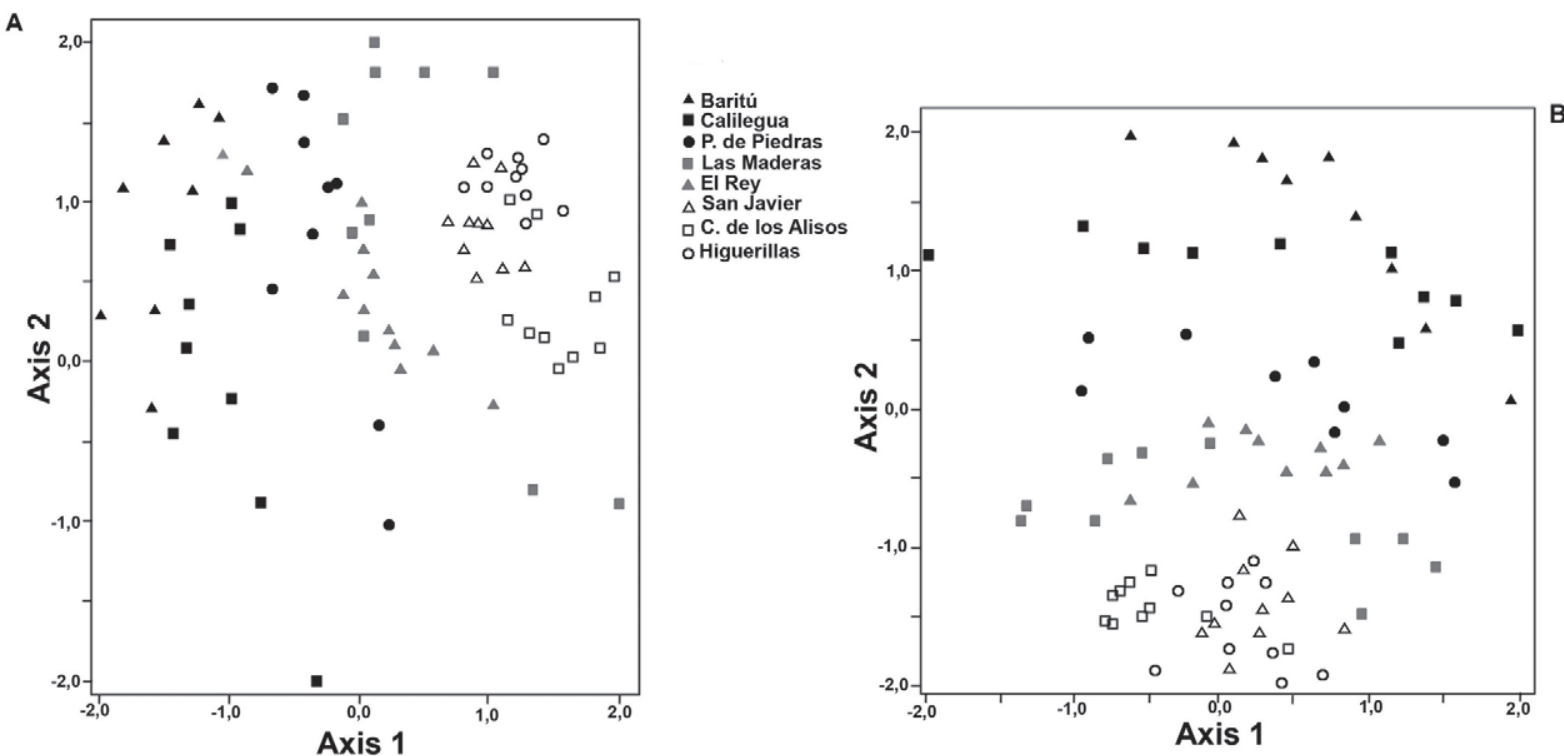

Figura 2. (A) Análisis multivariado de similitud Nonmetric Multidimensional Scaling entre la riqueza y los diferentes sitios de estudio de las selvas montanas de las Yungas de Argentina. (B) Análisis multivariado de similitud Nonmetric Multidimensional Scaling entre la abundancia relativa y los diferentes sitios de estudio de las selvas montanas de las Yungas de Argentina.

Figure 2. (A) Nonmetric Multidimensional Scaling multivariate similarity analysis between richness and the different study sites of the subtropical montane forests of the Yungas of Argentina. (B) Nonmetric Multidimensional Scaling multivariate similarity analysis between relative abundance and the different study sites of the subtropical montane forests of the Yungas of Argentina.

\section{DISCUSIÓN}

En las selvas montanas de las Yungas hay presentes aproximadamente 170 especies de aves del orden Passeriformes y de las familias Trochilidae y Picidae (Blendinger y Alvarez, 2009), que potencialmente podrían participar en una bandada mixta. En el presente estudio, 72 especies (42\%) fueron registradas participando. Valores similares fueron encontrados en otros sitios neotropicales de equivalentes condiciones. Por ejemplo, en Colombia, el $47 \%$ de las especies de la zona integraron bandadas mixtas (Guevara, Valarezo, Onofa, Cupuerán, 2011) y el 45\% en Bolivia (Martínez, 2003). Si bien en las Yungas de Argentina, habita un bajo número de especies en relación a otras selvas montanas del Neotrópico (Boyla y Estrada, 2005), el alto número de especies que se registró participando de las bandadas indicaría que es una importante característica de la avifauna de estas selvas.

Generalmente, dichas bandadas están conformadas por aves passeriformes, pero también participan especies de otros órdenes, como ser, Cuculiformes, Galbuliformes, Piciformes y Apodiformes (Munn, 1985). Por ejemplo, en Colombia están citados carpinteros como Piculus rubiginosus, P. rivolii y Veniliornis dignus (Arbalaéz-Cortés, Rodríguez-Correa, Restrepo-Chica, 2011), en Ecuador, Campephilus pollens (Guevara et al., 2011), en Perú, Picumnus rufiventris y Veniliornis passerinus (Munn 1985), en Bolivia, Piculus rivolli, Veniliomis nigriceps, Colaptes rubiginosus (Martínez 2003; Montaño-Centellas, 2020); o picaflores en Colombia como Coeligena coeligena y Agleiocercus kingi (Herrera Ordóñez, 2008). En nuestros sitios de estudio de Argentina encon- 
tramos bandadas mixtas conformadas por especies pertenecientes a tres órdenes: Passeriformes, Apodiformes (Trochilidae) y Piciformes (Picidae); aunque estos dos últimos órdenes sólo representaron el 12,5\% de las especies y tuvieron una baja ocurrencia. Esto nos podría estar indicando que las bandadas mixtas de las Yungas argentinas están compuestas casi totalmente por paseriformes, lo cual es coincidente con otros sitios neotropicales, indicando la gran preferencia de especies de este orden al conformar grupos mixtos (Munn y Terborgh, 1979; Jullien y Thiollay, 1998; Sridhar y Sankar, 2008; Montaño-Centellas, 2020).

En el presente estudio, las bandadas mixtas tuvieron una dominancia de Tyrannidae, Furnariidae y Thraupidae. Si bien, las dos primeras familias son importantes por su aporte de especies en algunas otras regiones neotropicales, principalmente de bosques y selvas tropicales (Munn y Terborgh, 1979; Poulsen 1996; Bohórquez, 2003; Martínez, 2003; Pomara et al., 2003), en la mayoría de las bandadas mixtas de dichos bosques y selvas, la familia más importante es la de los tráupidos (Martínez, 2003; Guevara et al., 2011). La causa por lo cual los tráupidos tuvieron comparativamente poca representación en las bandadas mixtas de las Yungas de Argentina, podría deberse a que la riqueza de especies de esta familia en Argentina es mucho menor que otras regiones neotropicales (106 especies), en comparación a Bolivia con 159 especies o Perú con 187 especies (Pearman y Areta 2020).

En comparación con otros trabajos realizados en el Neotrópico, tanto en bosques como en selvas tropicales y subtropicales, el número de bandadas mixtas observadas en el presente estudio fue intermedio. En proporción, el número de bandadas mixtas observadas en relación al esfuerzo de muestreo realizado, fue de 0,25 ; valor que está por encima al encontrado en los bosques montano de Polylepis de Bolivia $(0,16)$ (Herzog, Soria, Troncoso, Matthysen, 2002) y levemente por debajo de los observados para las selvas montanas de Bolivia (0,32) (Martínez, 2003) y Colombia $(0,32)$ (Arbeláez-Cortés et al., 2011). Si bien el rango en proporción fue de 0,16 a 0,78, en general los valores registrados fueron cercanos a 0,36 (Tabla 4). Esto significaría que los valores encontrados para las selvas montanas de las Yungas de Argentina se mantienen en el rango de otras selvas montanas neotropicales. El número total de especies participantes en bandadas mixtas (72) de nuestro estudio, resulta intermedio a lo encontrado en otras selvas y bosques neotropicales (Tabla 4). Donde se observa que participaron desde 27 especies en las selvas tropicales deciduas de México (Gram, 1998), hasta 114 en las selvas montanas de Bolivia (Montaño-Centellas, 2020). Cabe destacar que en algunos sitios se obtuvieron valores similares a los encontrados en el presente estudio. Por ejemplo, en la selva atlántica de Brasil y en los bosques montanos de Colombia participaron hasta 71 especies (Bohórquez, 2003; Maldonado-Coelho y Marini, 2004) y 75 en las selvas montanas de Colombia (Arbeláez-Cortés et al., 2011).

En promedio, las bandadas mixtas estudiadas estuvieron compuestas por 4,3 especies, valor intermedio a lo encontrado en otros lugares neotropicales (tropicales y subtropicales), que oscilaron entre 2,8 (bosques de Polylepis de Bolivia) y 10,3 (bosques montanos de Ecuador). Los valores de abundancia promedio registrados en el presente estudio $(6,9)$ fueron también intermedios en relación a los valores obtenidos en otras selvas y bosques neotropicales (19,3 en selvas tropicales de México y 5,4 en 
bosques de Polylepis de Bolivia) (Tabla 4). Esto nos indicaría que las características estructurales (número de especies y/o número de individuos participantes) de las bandadas mixtas tanto de las Yungas de Argentina, como la de otras selvas y bosques montanos neotropicales, es muy variable y no presentarían un patrón común general. La variabilidad en la participación podría estar dada por la diversidad de especies presente en un sitio determinado más que por patrones generales (Goodale et al., 2020).

Como fuera indicado por Latta y Wunderle (1996), la riqueza de especies y el número de individuos presentes en las bandadas mixtas, son un reflejo del pool de especies presentes en un hábitat determinado. Como este estudio, que se realizó a lo largo de un gradiente latitudinal, tanto del clima como de la vegetación, el pool de especies de aves fue diferente en los sitios estudiados, lo cual influyó en la composición de las bandadas mixtas, ya que se encontró una notable similitud entre los sitios pertenecientes al mismo sector y una disimilitud entre los diferentes sectores. Algunos autores (Bohórquez, 2003; Goodale et al., 2010; Colorado y Rodewald, 2015) señalaron que las bandadas mixtas generalmente, no se conforman con especies congenéricas simultáneamente, evitando de esta forma la competencia intra-género e interespecífica. Entre los sectores de las Yungas argentinas se pudo observar claramente este patrón. Por ejemplo, mientras que algunas especies están fuertemente asociadas o son exclusivas del sector Norte-Centro (Myiothlypis (=Basileuterus) bivittata, Arremon (=Atlapetes) torquatus y Atlapetes fulviceps), otras lo son del sector Sur (Basileuterus culicivorus y Atlapetes citrinellus); estas especies presentarían una similitud filogenética que les generaría la misma predisposición a formar parte de bandadas mixtas (Goodale et al. 2020). La separación en la distribución de las especies del género Myiothlypis (=Basileuterus) en las Yungas de Argentina ya había sido indicado por Capllonch (2007).

Si bien el gradiente latitudinal no tuvo influencia directa sobre el número, riqueza y abundancia de las bandadas mixtas analizándolas de manera independiente, se observó que, al analizar las interacciones entre las variables estructurales, sí hubo relación. El número de especies participantes sí estuvo relacionado al número de bandadas mixtas, es decir, cuantas más bandadas se formen en un lugar, más especies podrían participar. A su vez, el número de bandadas mixtas estaría relacionado con el número de individuos participantes, es decir, a mayor número de bandadas más individuos participarían. Además la abundancia de individuos en las bandadas se relacionó de manera inversa con la riqueza, por lo que podríamos decir que bandadas de gran participación de individuos serían conformadas por pocas especies, y esto podría deberse a las características distintivas de ciertas especies a la agregación. Sin embargo, cabe destacar que dichas interacciones observadas estarían dadas por cuestiones locales (influenciadas por el clima local y/o vegetación) de cada sitio y no influenciadas por el gradiente latitudinal de las Yungas en general. No obstante, se pudo observar que la composición de especies en las bandadas mixtas sí varió a lo largo del gradiente y esto podría deberse a la distribución propia de varias especies y a la mayor predisposición de algunas especies a participar dentro de una bandada. Estudios previos, evidenciaron que la variación en la estructura y composición de las bandadas mixtas puede deberse a diferentes factores, tanto locales como regionales, 
Tabla 4. Número de bandadas mixtas observadas, entre paréntesis el tiempo total de observación. Número total de especies, número promedio de especies y de individuos en bandadas mixtas en diferentes bosques y selvas neotropicales

Table 4. Number of mixed-species flocks observed, total observation time in parentheses. Total number of species, average number of species and individuals in mixed-species flocks in different Neotropical forest.

\begin{tabular}{|c|c|c|c|c|c|c|}
\hline Ambiente de estudio & $\begin{array}{c}\mathrm{N}^{\circ} \text { de } \\
\text { bandadas } \\
\text { mixtas } \\
\text { ( } \mathrm{n}^{\circ} \text { de hs. } \\
\text { mues- } \\
\text { treadas) }\end{array}$ & $\begin{array}{c}\text { Propoción } \\
\text { de } \mathrm{N}^{\circ} \text { de } \\
\text { bandadas } \\
\text { mixtas } \\
\text { según } \\
\text { esfuerzo de } \\
\text { muestreo }\end{array}$ & $\begin{array}{l}N^{\circ} \text { total } \\
\text { de } \\
\text { especies }\end{array}$ & $\begin{array}{l}\mathrm{N}^{\circ} \text { prome- } \\
\text { dio de } \\
\text { especies }\end{array}$ & $\begin{array}{c}N^{\circ} \text { prome- } \\
\text { dio de } \\
\text { individuos }\end{array}$ & Fuente \\
\hline Selvas Tropicales Deciduas (México) & $24(120)$ & 0,20 & 27 & 6,5 & 10,6 & Gram, 1998 \\
\hline Selva Tropicales (México) & $42(120)$ & 0,35 & 39 & 9,3 & 19,3 & Gram, 1998 \\
\hline $\begin{array}{l}\text { Bosques Nublados } \\
\text { (Rep. Dominicana y Haití) }\end{array}$ & $180(232)$ & 0,78 & 46 & 7,1 & 11,3 & Latta y Wunderle, 1996 \\
\hline Selva Atlántica (Brasil) & $356(960)$ & 0,37 & 71 & 6,0 & 10,9 & $\begin{array}{l}\text { Maldonado-Coelho y } \\
\text { Marini, } 2004\end{array}$ \\
\hline Bosque Montano (Colombia) & $184(576)$ & 0,32 & 71 & 5,8 & 8,3 & Bohórquez, 2003 \\
\hline Bosque Montano (Colombia) & $64(121)$ & 0,53 & 42 & 5,1 & 11,5 & $\begin{array}{c}\text { Arbeláez-Cortés } \\
\text { y Marín-Gómez, } 2012\end{array}$ \\
\hline Selva Montana (Colombia) & $104(324)$ & 0,32 & 75 & 9,4 & --- & $\begin{array}{l}\text { Arbeláez-Cortés et al., } \\
2011\end{array}$ \\
\hline Bosque Montano (Ecuador) & $53(150)$ & 0,35 & 112 & 10,3 & 16,6 & Guevara et al., 2011 \\
\hline Bosques de Polylepis (Bolivia) & $53(320)$ & 0,16 & 10 & 2,8 & 5,4 & Herzog et al., 2002 \\
\hline Selva Montana (Bolivia) & $57(180)$ & 0,32 & 33 & 4,4 & 12,3 & Martínez, 2003 \\
\hline Selva Montana de Yungas & $325(1280)$ & 0,25 & 72 & 4,3 & 6,9 & Presente estudio \\
\hline
\end{tabular}

como los diferentes gradientes donde se desplacen (por ejemplo, gradientes altitudinales), las características locales de la vegetación, las condiciones de conservación de un paisaje o las condiciones climáticas locales (Mokross et al., 2014; Goodale et al., 2015; Kajiki. et al., 2018; Zarco et al. 2019; Montaño-Centellas, 2020; Jones, Walters, Robinson, 2021; Montaño-Centellas y Jones, 2021).

Este es el primer estudio de estructura y composición de las especies de bandadas mixtas que abarca las selvas montanas de las Yungas de Argentina. Se espera que el mismo sea de utilidad en futuros estudios de bandadas para comparar con otros sitios de Argentina y del Neotrópico; para evaluar variaciones en el tiempo y en otros pisos altitudinales de las Yungas y para determinar si existe influencia de los disturbios y modificaciones en la vegetación sobre las mismas.

\section{AGRADECIMIENTOS}

A Administración de Parques Nacionales Seccional NOA, Administración del Parque Sierra de San Javier (UNT) y al Sr. Mauricio Flores por permitirnos el ingreso a los sitios de estudio. A Manuel Nores, John Blake, Peter Feisinger, y revisores anónimos por sus valiosos aportes. A nuestros asistentes de campo. A Pablo Pereyra por la colaboración en la elaboración del mapa. 


\section{FINANCIAMIENTO}

El presente trabajo fue financiado por CONICET y Fundación Miguel Lillo.

\section{PARTICIPACIÓN}

MEF realizó el muestreo de campo, análisis de datos y redacción del manuscrito. ALE y MVM participaron en el análisis de datos y redacción del manuscrito.

\section{CONFLICTO DE INTERÉS}

No existe conflicto de interés entre autores o con terceros.

\section{LITERATURA CITADA}

Amaral, P.P., Ragusa-Netto, J. (2008). Bird mixed-flocks and nuclear species in a Tecoma savanna in the Pantanal. Brazilian Journal of Biology, 68, 511-518.

Arbeláez-Cortés, E., Rodríguez-Correa, H.A., Restrepo-Chica, M. (2011). Mixed bird flocks: patterns of activity and species composition in a region of the Central Andes of Colombia. Revista Mexicana de Biodiversidad, 82, 639-651.

Bibby, C., Jones, M., Marsden, S. (2000). Expedition field techniques. Bird surveys. Ed BirdLife International. UK.

Blendinger, P.G., Alvarez, M.E. (2009). Aves de la Selva Pedemontana. En Selva Pedemontana de las Yungas: historia natural, ecología y manejo de un ecosistema en peligro (233-272). Ediciones del Subtrópico, Yerba Buena, Tucumán.

Blundo, C., Malizia, L.R., Blake, J.G., Brown, A.D. (2012). Tree species distribution in Andean forests: influence of regional and local factors. Journal of Tropical Ecology, 28, 83-95.

Bohórquez, C.I. (2003). Mixed-species bird flocks in a montane cloud forest of Colombia. Ornitolgía Neotropropical, 14, 67-78.

Borah, B., Quader, S., Srinivasan, U. (2018). Responses of interspecific associations in mixed-species bird flocks to selective logging. Journal of Applied Ecology, 55, 1637-1646.

Boyla, K., Estrada, A. (2005). Áreas Importantes para la Conservación de las Aves en los Andes Tropicales: sitios prioritarios para la conservación de la biodiversidad. BirdLife International. Serie de Conservación 14, Quito.

Brown, A.D., Grau, H.R., Malizia, L.R., Grau, A. (2001). Argentina. En Bosques Nublados del Neotrópico (623-659). Instituto Nacional de Biodiversidad, INBio. Santo Domingo de Heredia, Costa Rica.

Cabrera, A.L. (1976). Regiones fitogeográficas de la Argentina. Enciclopedia Argentina de Agricultura y Ganadería 2, 1-85.

Capllonch, P. (2007). Distribución latitudinal y altitudinal de tres especies del género Basileuterus en el Noroeste argentino. Hornero, 22, 23-28.

Colorado G.J. (2013). Why animals come together, with the special case of mixed-species bird flocks. Revista EIA Escuela de Ingeniería de Antioquia, 10, 49-66. 
Colorado, G.J., Rodewald, A.D. (2015). Response of mixed-species flocks of specialist insectivore birds to habitat alteration and deforestation in the Andes. Biology Conservation, 188, 72-81.

Cordeiro, N.J., Borghesio, L., Joho, M.P., Monoski, T.J., Mkongewa, V.J., Dampf, C.J. (2015). Forest fragmentation in an African biodiversity hotspot impacts mixed-species bird flocks. Biology Conservation, 188, 61-71.

Fanjul, M.E., Echevarria, A.L. (2015). Composición, estructura y rol social de las bandadas mixtas de aves de la selva montana de Yungas, provincia de Tucumán, Argentina. Acta Zoológica Lilloana, 59, 141-154.

Goodale, E., Beauchamp, G., Magrath, R.D., Nieh, J.C., Ruxton, G.D. (2010). Interspecific information transfer influences animal community structure. Trends in Ecology \& Evolution, 25, 354-361.

Goodale, E., Ding, P., Liu, X., Martínez, A., Si, X., Walters, M., Robinson, S.K. (2015). The structure of mixed-species bird flocks, and their response to anthropogenic disturbance, with special reference to East Asia. Avian Research, 6, 14.

Goodale, E., Sridhar, H., Sieving, K.E., Bangal, P., Colorado Z., G.J., Farine, D.R., Heymann, E.W., Jones, H.H., Krams, I., Martínez, A.E., Montaño-Centellas, F., Muñoz, J., Srinivasan, U., Theo, A., Shanker, K. (2020). Mixed company: a framework for understanding the composition and organization of mixed-species animal groups. Biological Reviews, 95, 889-910.

Gram, W.K. 1998. Winter participation by neotropical migrant and resident birds in mixed-species flocks in northeastern Mexico. Condor, 100, 44-53.

Guevara, E.A., Valarezo, J.C., Onofa, A., Cupuerán, F. (2011). Mixed-species flock composition in a Northwestern Ecuadorian cloud forest. Ornitología Neotropical, 22, 379-386.

Herrera Ordóñez, R. (2008). Composición de especies y variación estacional de bandadas mixtas de aves en dos tipos de bosques en la Cordillera Oriental de Colombia. Tesis de Licenciatura. Universidad Industrial de Santander.

Herzog, S.K., Soria, R., Troncoso, A., Matthysen, E. (2002). Composition and structure of avian mixed-species flocks in a High-Andean Polylepis forest in Bolivia. Ecotropica, 8, 133-143.

Hutto, R.L. (1987). A description of mixed-species insectivorous bird flocks in Western Mexico. Condor, 89, 282-292.

Ippi, S., Trejo, A. (2003). Dinámica y estructura de bandadas mixtas de aves en un bosque de Lenga (Nothofagus pumilio) del noroeste de la Patagonia Argentina. Ornitología Neotropical, 14, 353-362.

Jones, H.H., Walters, M.J., Robinson, S.K. (2021). Effect of temperature on flocking propensity and species interactions in a subtropical mixed-species flocking system. The Wilson Journal of Ornithology, 132, 560-574.

Jullien, M., Thiollay, J.M. (1998). Multi-species territoriality and dynamic of neotropical forest understorey bird flocks. Journal of Animal Ecology, 67, 227-252.

Kajiki, L.N; Montaño-Centellas, F., Mangini, G., Colorado Z., G.J., Fanjul, M.E. (2018). Ecology of mixed-species flocks of birds across gradients in the Neotropics. Revista Brasileira de Ornitología, 26, 82-89. 
King, D.I., Rappole, J.H. (2000). Winter flocking of insectivorous birds in Montane Pine-Oak Forests in Middle America. Condor, 102, 664-672.

Knowlton, J.L., Graham, C.H. (2011). Species interactions are disrupted by habitat degradation in the highly threatened Tumbesian region of Ecuador. Ecological Applications, 21, 2974-2986.

Latta, S.C., Wunderle, J.M. (1996). Ecological relationships of two todies in Hispaniola: Effects of habitat and flocking. Condor, 98, 769-779.

Magurran, A.E. (1989). Diversidad Ecológica y su Medición. Ed. Vedrà. Barcelona, España.

Maldonado-Coelho, M., Marini, M. Â. (2000). Effects of forest fragment size and successional stage on mixed-species bird flocks in southeastern Brazil. Condor, $102,585-594$.

Maldonado-Coelho, M., Marini, M.A. (2004). Mixed-species bird flocks from Brazilian Atlantic forest: the effects of forest fragmentation and seasonality on their size, richness and stability. Biological Conservation, 116, 19-26.

Malizia, L., Pacheco, S., Blundo, C., Brown, A.D. 2012. Caracterización altitudinal, uso y conservación de las Yungas Subtropicales de Argentina. Ecosistemas 21: 53-73.

Mangini, G, Fanjul, M.E. (2013). Conociendo las bandadas mixtas de aves y los efectos de la fragmentación en bosques y selvas de la provincia de Salta. Temas de Biología y Geología del Noa, 3, 68-76.

Mangini, G.G., Areta, J.I. (2018). Bird Mixed-Species Flock Formation Is Driven by Low Temperatures between and within Seasons in a Subtropical AndeanFoothill Forest. Biotropica, 50, 816-825.

Martínez, O. (2003). Composición por especies y uso de sustratos por las bandadas mixtas de aves en un bosque nublado andino de Bolivia. Ecología de Bolivia, $38,99-119$.

McCune, B., Grace, J.B. (2002). Analysis of ecological communities. MjM Software Design, Gleneden Beach, Oregon, USA.

Mokross, K., Ryder, T.B., Côrtes, M.C., Wolfe, J.D., Stouffer, P.C. (2014). Decay of interspecific avian flock networks along a disturbance gradient in Amazonia. Proceedings of the Royal Society B, 281, 20132599.

Montaño-Centellas, F.A. (2020). Interaction networks of avian mixed-species flocks along elevation in the tropical Andes. Ecography, 43, 930-942.

Montaño-Centellas, F.A., Jones, H.H. (2021). Temperature and vegetation complexity structure mixed-species flocks along a gradient of elevation in the tropical Andes. Ornithology, 138, 1-18.

Morales, J.M., Sirombra, M., Brown, A.D. (1995). Riqueza de árboles en las Yungas Argentinas. En Investigación, Conservación y Desarrollo en Selvas Subtropicales de Montaña (163-174). Proyecto de Desarrollo Forestal, LIEY, Univ. Nacional de Tucumán.

Morse, D.H. (1970). Ecological Aspects of some Mixed-Species Foraging Flocks of Birds. Ecological Monographs, 40, 119-168.

Morse, D.H. (1977). Feeding behavior and predator avoidance in heterospecific groups. Bioscience, 27, 332-339. 
Moynihan, M. (1962). The organization and probable evolution of some mixed species flocks of neotropical birds. Smithsonian Miscellaneous Collections, 143, 1-140.

Munn, C.A. (1983). Densidad de población, ecología y comportamiento de bandadas mixtas en la selva baja del Parque Nacional Manu, Madre de Dios, Perú. En: $1^{\circ}$ Simposio de Ornitología Neotropical. Pp. 49-54. Arequipa, Perú.

Munn, C.A. (1985). Permanent canopy and understory flocks in Amazonia: species composition and population density. En Neotropical ornithology (683-712). Ornithological Monographs 36. American Ornithologists' Union, Washington, D.C., USA.

Munn, C.A., Terborgh, J.W. (1979). Multi-species territoriality in neotropical foraging flocks. Condor, 81, 338-347.

Nores, M. (1989). Patrones de distribución y causa de especiación en aves argentinas. Tesis Doctoral. Univ. Nacional de Córdoba.

Ojeda, R.A, Barquez, R.M., Stadler, J., Brandl, R. (2008). Decline of mammal species diversity along the Yungas forest of Argentina. Biotropica, 40, 515-521.

Pearman, M., Areta, J.J. (2020). Species lists of birds for South American countries and territories: Argentina. [Version 17/09/2021]. http://www.museum.lsu. edu/ Remsen/SACCCountryLists.htm

Pomara, L.Y., Cooper, R.J., Petit, L. J. (2003). Mixed-species flocking and foraging behavior of four neotropical warblers in Panamanian shade coffee fields and forests. Auk, 120, 1000-1012.

Poulsen, B.O. (1996). Species composition, function and home-range of mixed-species bird flocks in a primary cloud forest in Ecuador. Bull. Br. Ornithology Club, 116, 67-74.

Powell, G.V.N. (1985). Sociobiology and Adaptive Significance of Interspecific Foraging Flocks in the Neotropics. En Neotropical ornithology (713-732). Ornithological Monographs 36. American Ornithologists' Union, Washington, D.C., USA.

R Development Core Team. 2004. R: a language and environment for statistical computing. R Foundation for Statistical Computing, Vienna, Austria URL: http://www.R-project.org. ISBN3-900051-00-3

Ralph, C.J., Geupel, G.R., Pyle, P., Martin, T.E., DeSante, D.F., Milá, B. (1996). Manual de Método de Campo para el Monitoreo de Aves Terrestres. Gen. Tech. Rep. PSW-GTR-1959. Albany. CA: Pacific Southwest Research Station, Forest Service, U.S. Dep. of Agric. 44 pp.

Sazima, C., Krajewski, J.P., Bonaldo, R.M., Sazima, I. (2007). Nuclear-follower foraging associations of reef fishes and other animals at an oceanic archipelago. Environmental Biology of Fishes, 80, 351-361.

Semeniuk, C.A.D., Dill, L.M. (2006). Anti-predator benefits of mixed species groups of cowtail stingrays (Pastinachus sephen) and whit prays (Himantura uarnak) at rest. Ethology, 112, 33-43.

Sridhar, H., Sankar, K. (2008). Effects of habitat degradation on mixed-species bird flocks in Indian Rain Forests. Journal of Tropical Ecology, 24, 135-147.

Sridhar, H., Srinivasan, U., Askins, R.A., Canales-Delgadillo, J.C., Chen, C., Ewert, D.N., Gale, G.A., Goodale, E., Gram, W.K., Hart, P.J., Hobson, K.A., Hutto, 
R.L., Kotagama, S.W., Knowlton, J.L., Lee, T.M., Munn, C.A., Nimnuan, S., Nizam, B.Z., Péron, G., Robin, V.V., Rodewald, A.D., Rodewald, P.G., Thomson, R.L., Trivedi, P., Van Wilgenburg, S.L., Shanker, K. (2012). Positive relationships between association strength and phenotypic similarity characterize the assembly of mixed-species bird flocks worldwide. The American Naturalist, $180,777-790$.

Stensland, E., Angerbjörn, A., Berggren, P. (2003). Mixed Species groups in mammals. Mammal Review, 33, 205-223.

Stojan-Dólar, M., Heymann, E.W. (2010). Vigilance of mustached tamarins in single-species and mixed-species groups, the influence of group composition. Behavioural Ecology Sociobiology, 64, 325-335.

Struhsaker, T.T. (1981). Polyspecific associations among tropical rainforest primates. Zeitschrift Für Tierpsychologie, 57, 268-304.

Terborgh, J. (1990). Mixed flocks and polyspecific associations: costs and benefits of mixed groups to birds and monkeys. American Journal of Primatology, 21, 87-100.

Tierno de Figueroa, J.M., Padial, J.M. (2005). Avifauna invernal en ambientes de Chaco y Pantanal en Bahía Negra (norte de Paraguay). Hornero, 20, 153-162.

Tubelis, D.P., Cowling, A., Donnelley, C. (2006). Role of mixed-species flocks in the use of adjacent savannas by forest birds in the central Cerrado, Brazil. Austral Ecology, 31, 38-45.

Tubelis, D.P. (2007). Mixed-species flocks of birds in the Cerrado, South America: a review. Ornitología Neotropical, 18, 75-97.

Vervoorst, F. (1979). La vegetación del Noroeste Argentino y su degradación. Serie Conservación de la Naturaleza, Fundación Miguel Lillo, Tucumán, Argentina. 9 pp.

Vides-Almonacid, R. (1993). Estudio comparativo de la taxocenosis de aves de bosques montanos de las Sierras de San Javier, Tucumán, Argentina: bases para su manejo y conservación. Tesis Doctoral. Univ. Nacional de Tucumán.

Zarco, A., Cueto V.R. (2017). Winter flock structure in the central Monte desert, Argentina. Ardea, 105, 89-97.

Zarco, A., Cueto, V.R., Sagario, M.C., Marone, L. (2019). Effects of livestock grazing on flocks of seed-eating birds in the central Monte desert, Argentina. Canadian Journal of Zoology, 97, 606-611.

Zhang, Q., Han, R., Huang, Z., Zou, F. (2013). Linking vegetation structure and bird organization: response of mixed-species bird flocks to forest succession in subtropical China. Biodiversity and Conservation, 22, 1965-1989.

Zou, F., Jones, H., Colorado Z., G.J., Jiang, D., Lee, T.M., Martínez, A., Sieving, K., Zhang, M., Zhang, Q., Goodale, E. (2018). The conservation implications of mixed-species flocking in terrestrial birds, a globally-distributed species interaction network. Biological Conservation, 224, 267-276.

Zuur, A.F., Ieno, E.N., Walker, N.J., Saveliev, A.A., Smith, G.M. (2009). Mixed effects models and extensions in ecology with R. Springer 
Apéndice 1 (hoja 1 de 2). Presencia-Ausencia y Ocurrencia (\%) de las especies que conforman las Bandadas Mixtas en cada sitio de estudio.

Appendix 1 (page 1 of 2). Presence-Absence and Occurrence (\%) of the species that make up the Mixed Flocks at each study site.

\begin{tabular}{|c|c|c|c|c|c|c|c|c|c|}
\hline Especies & Baritú & Calilegua & $\begin{array}{l}\text { P. de } \\
\text { Piedras }\end{array}$ & $\begin{array}{c}\text { Las } \\
\text { Maderas }\end{array}$ & El Rey & $\begin{array}{l}\text { San } \\
\text { Javier }\end{array}$ & Alisos & $\begin{array}{l}\text { Higue- } \\
\text { rillas }\end{array}$ & $\begin{array}{l}\text { Ocurren } \\
\text { cia (\%) }\end{array}$ \\
\hline \multicolumn{10}{|l|}{ Trochilidae } \\
\hline Elliotomyia chionogaster & 0 & 0 & 0 & 1 & 0 & 1 & 0 & 1 & 0,92 \\
\hline Chlorostilbon lucidus & 1 & 1 & 1 & 0 & 0 & 0 & 0 & 0 & 1,23 \\
\hline Sappho sparganurus & 0 & 1 & 1 & 0 & 0 & 0 & 1 & 1 & 2,15 \\
\hline \multicolumn{10}{|l|}{ Picidae } \\
\hline Campephilus leucopogon & 0 & 0 & 0 & 0 & 0 & 0 & 1 & 1 & 0,62 \\
\hline Dryobates fumigatus & 1 & 0 & 1 & 0 & 0 & 0 & 0 & 0 & 0,62 \\
\hline Dryobates frontalis & 1 & 0 & 1 & 1 & 1 & 1 & 0 & 1 & 4,62 \\
\hline Picumnus cirratus & 1 & 1 & 1 & 1 & 1 & 1 & 1 & 0 & 5,23 \\
\hline Colaptes rubiginosus & 0 & 1 & 0 & 1 & 1 & 0 & 0 & 1 & 3,08 \\
\hline \multicolumn{10}{|l|}{ Furnariidae } \\
\hline Dendrocolaptes picumnus & 0 & 0 & 1 & 1 & 0 & 0 & 0 & 0 & 0,92 \\
\hline Xiphocolaptes major & 0 & 0 & 1 & 0 & 0 & 0 & 0 & 0 & 0,31 \\
\hline Lepidocolaptes angustirostris & 0 & 0 & 1 & 1 & 0 & 0 & 0 & 0 & 1,23 \\
\hline Sittasomus griseicapillus & 1 & 1 & 1 & 1 & 1 & 1 & 1 & 1 & 10,15 \\
\hline Synallaxis frontalis & 0 & 0 & 0 & 1 & 0 & 0 & 1 & 0 & 0,92 \\
\hline Synallaxis azarae & 1 & 1 & 1 & 1 & 1 & 1 & 1 & 1 & 10,15 \\
\hline Synallaxis albescens & 0 & 0 & 0 & 1 & 0 & 0 & 0 & 0 & 0,31 \\
\hline Phacellodomus rufifrons & 1 & 0 & 1 & 1 & 0 & 0 & 0 & 0 & 1,23 \\
\hline Syndactyla rufosuperciliata & 1 & 1 & 1 & 1 & 1 & 1 & 1 & 1 & 53,23 \\
\hline Xenops rutilans & 1 & 0 & 1 & 1 & 0 & 0 & 0 & 0 & 1,54 \\
\hline \multicolumn{10}{|l|}{ Thamnophilidae } \\
\hline Batara cinerea & 0 & 1 & 0 & 0 & 0 & 0 & 0 & 0 & 0,31 \\
\hline Thamnophilus ruficapillus & 0 & 0 & 1 & 0 & 0 & 0 & 0 & 0 & 1,54 \\
\hline Thamnophilus caerulescens & 6 & 1 & 1 & 0 & 1 & 0 & 0 & 0 & 2,46 \\
\hline \multicolumn{10}{|l|}{ Tityridae } \\
\hline Pachyramphus polychopterus & 0 & 0 & 1 & 0 & 0 & 0 & 0 & 1 & 0,62 \\
\hline Pachyramphus viridis & 0 & 0 & 0 & 0 & 0 & 0 & 0 & 1 & 0,31 \\
\hline \multicolumn{10}{|l|}{ Tyrannidae } \\
\hline Ochthoeca leucophrys & 0 & 0 & 0 & 0 & 0 & 0 & 0 & 1 & 0,31 \\
\hline Lathrotriccus euleri & 0 & 0 & 1 & 0 & 0 & 0 & 0 & 0 & 0,31 \\
\hline Tolmomyias sulphurescens & 1 & 1 & 1 & 0 & 1 & 0 & 0 & 1 & 2,46 \\
\hline Poecilotriccus plumbeiceps & 0 & 1 & 1 & 1 & 1 & 0 & 0 & 0 & 4,62 \\
\hline Phylloscartes ventralis & 1 & 1 & 1 & 1 & 1 & 1 & 1 & 1 & 50,15 \\
\hline Serpophaga munda & 0 & 0 & 0 & 1 & 0 & 0 & 0 & 0 & 0,31 \\
\hline Mecocerculus leucophrys & 1 & 1 & 1 & 1 & 1 & 1 & 1 & 1 & 16,61 \\
\hline Elaenia obscura & 2 & 0 & 0 & 0 & 0 & 0 & 0 & 0 & 0,62 \\
\hline Elaenia parvirostris & 0 & 0 & 0 & 0 & 0 & 1 & 0 & 0 & 0,62 \\
\hline Elaenia strepera & 0 & 0 & 0 & 1 & 0 & 0 & 0 & 0 & 0,31 \\
\hline Phaeomyias murina & 0 & 0 & 0 & 1 & 0 & 0 & 0 & 1 & 1,23 \\
\hline Camptostoma obsoletum & 0 & 0 & 0 & 0 & 0 & 0 & 1 & 0 & 0,31 \\
\hline \multicolumn{10}{|l|}{ Corvidae } \\
\hline Cyanocorax chrysops & 0 & 0 & 0 & 1 & 0 & 0 & 0 & 1 & 1,23 \\
\hline
\end{tabular}


Apéndice 1 (hoja 2 de 2). Presencia-Ausencia y Ocurrencia (\%) de las especies que conforman las Bandadas Mixtas en cada sitio de estudio.

Appendix 1 (page 2 of 2). Presence-Absence and Occurrence (\%) of the species that make up the Mixed Flocks at each study site.

\begin{tabular}{|c|c|c|c|c|c|c|c|c|c|}
\hline Especies & Baritú & Calilegua & $\begin{array}{l}\text { P. de } \\
\text { Piedras }\end{array}$ & $\begin{array}{c}\text { Las } \\
\text { Maderas }\end{array}$ & El Rey & $\begin{array}{c}\text { San } \\
\text { Javier }\end{array}$ & Alisos & $\begin{array}{l}\text { Higue- } \\
\text { rillas }\end{array}$ & $\begin{array}{l}\text { Ocurren- } \\
\text { cia (\%) }\end{array}$ \\
\hline \multicolumn{10}{|l|}{ Troglodytidae } \\
\hline Troglodytes aedon & 1 & 1 & 1 & 1 & 0 & 1 & 1 & 1 & 7,38 \\
\hline Troglodytes solstitialis & 0 & 1 & 1 & 1 & 1 & 0 & 1 & 0 & 3,38 \\
\hline \multicolumn{10}{|l|}{ Turdidae } \\
\hline Catharus dryas & 0 & 1 & 0 & 0 & 0 & 0 & 0 & 0 & 0,31 \\
\hline Turdus nigriceps & 0 & 1 & 0 & 0 & 1 & 1 & 0 & 0 & 3,38 \\
\hline Turdus chiguanco & 0 & 1 & 0 & 0 & 0 & 0 & 0 & 0 & 0,62 \\
\hline Turdus rufiventris & 1 & 1 & 1 & 1 & 1 & 1 & 1 & 1 & 20,31 \\
\hline Turdus serranus & 0 & 1 & 0 & 0 & 0 & 0 & 0 & 0 & 0,31 \\
\hline \multicolumn{10}{|l|}{ Vireonidae } \\
\hline Cyclarhis gujanensis & 1 & 1 & 1 & 1 & 1 & 0 & 1 & 0 & 3,08 \\
\hline \multicolumn{10}{|l|}{ Parulidae } \\
\hline Myiothlypis signata & 1 & 1 & 0 & 0 & 1 & 0 & 0 & 0 & 2,15 \\
\hline Myiothlypis bivittata & 1 & 1 & 1 & 1 & 1 & 0 & 0 & 0 & 26,77 \\
\hline Basileuterus culicivorus & 0 & 1 & 0 & 0 & 1 & 1 & 1 & 1 & 12,92 \\
\hline Myioborus brunniceps & 1 & 1 & 1 & 1 & 1 & 1 & 1 & 1 & 35,39 \\
\hline Setophaga pitiayumi & 1 & 1 & 1 & 1 & 1 & 1 & 1 & 1 & 26,15 \\
\hline \multicolumn{10}{|l|}{ Fringillidae } \\
\hline Spinus magellanicus & 0 & 0 & 0 & 0 & 0 & 0 & 1 & 0 & 0,31 \\
\hline Chlorophonia cyanocephala & 0 & 0 & 0 & 0 & 0 & 1 & 1 & 1 & 1,23 \\
\hline Euphonia chlorotica & 0 & 0 & 0 & 0 & 0 & 1 & 0 & 1 & 0,92 \\
\hline \multicolumn{10}{|l|}{ Thraupidae } \\
\hline Thlypopsis sordida & 0 & 0 & 0 & 0 & 0 & 1 & 0 & 0 & 0,62 \\
\hline Thlypopsis ruficeps & 0 & 1 & 0 & 0 & 0 & 0 & 0 & 0 & 0,31 \\
\hline Thraupis sayaca & 1 & 1 & 1 & 1 & 1 & 1 & 1 & 1 & 14,15 \\
\hline Rauenia bonariensis & 0 & 0 & 0 & 0 & 1 & 1 & 0 & 0 & 0,62 \\
\hline Pipraeidea melanonota & 0 & 0 & 0 & 0 & 0 & 1 & 0 & 1 & 3,08 \\
\hline Sicalis flaveola & 0 & 0 & 0 & 0 & 0 & 0 & 0 & 1 & 0,62 \\
\hline Microspingus erythrophrys & 1 & 1 & 1 & 1 & 1 & 1 & 1 & 1 & 10,46 \\
\hline Microspingus melanoleuca & 0 & 0 & 0 & 1 & 0 & 0 & 0 & 1 & 0,92 \\
\hline Saltator aurantiirostris & 1 & 0 & 1 & 1 & 1 & 0 & 0 & 1 & 3,38 \\
\hline \multicolumn{10}{|l|}{ Cardinalidae } \\
\hline Piranga flava & 0 & 0 & 0 & 1 & 1 & 0 & 0 & 1 & 1,23 \\
\hline Pheucticus aureoventris & 1 & 0 & 0 & 1 & 0 & 1 & 0 & 1 & 3,69 \\
\hline Cyanoloxia brissonii & 0 & 0 & 1 & 1 & 0 & 0 & 0 & 0 & 1,23 \\
\hline \multicolumn{10}{|l|}{ Passerellidae } \\
\hline Arremon flavirostris & 1 & 1 & 1 & 1 & 1 & 1 & 1 & 1 & 21,23 \\
\hline Arremon torquatus & 1 & 1 & 1 & 1 & 1 & 0 & 0 & 0 & 16,92 \\
\hline Atlapetes citrinellus & 0 & 0 & 0 & 0 & 1 & 1 & 1 & 1 & 6,77 \\
\hline Atlapletes fulviceps & 0 & 1 & 0 & 0 & 0 & 0 & 0 & 0 & 0,62 \\
\hline Chlorospingus flavopectus & 1 & 1 & 1 & 1 & 1 & 1 & 1 & 1 & 50,15 \\
\hline \multicolumn{10}{|l|}{ Icteridae } \\
\hline Cacicus chrysopterus & 0 & 1 & 1 & 1 & 1 & 0 & 0 & 1 & 4,00 \\
\hline Icterus pyrrhopterus & 0 & 0 & 0 & 1 & 0 & 0 & 0 & 0 & 0,62 \\
\hline
\end{tabular}

